\title{
Collaborators Awareness for User Cohabitation in Co-located Collaborative Virtual Environments
}

\author{
Jérémy Lacoche \\ IRT $b<>$ com \\ Rennes, France \\ jeremy.lacoche@b-com.com \\ Thomas Boggini \\ IRT $b<>$ com \\ Rennes, France \\ thomas.boggini@b-com.com
}

\author{
Nico Pallamin \\ IRT $b<>$ com \\ Rennes, France \\ nico.pallamin@b-com.com \\ Jérôme Royan \\ IRT $\mathrm{b}<>$ com \\ Rennes, France \\ jerome.royan@b-com.com
}

\begin{abstract}
In a co-located collaborative virtual environment, multiple users share the same physical tracked space and the same virtual workspace. When the virtual workspace is larger than the real workspace, navigation interaction techniques must be deployed to let the users explore the entire virtual environment. When a user navigates in the virtual space while remaining static in the real space, his/her position in the physical workspace and in the virtual workspace are no longer the same. Thus, in the context where each user is immersed in the virtual environment with a Head-Mounted-Display, a user can still perceive where his/her collaborators are in the virtual environment but not where they are in real world. In this paper, we propose and compare three methods to warn users about the position of collaborators in the shared physical workspace to ensure a proper cohabitation and safety of the collaborators. The first one is based on a virtual grid shaped as a cylinder, the second one is based on a ghost representation of the user and the last one displays the physical safe-navigation space on the floor of the virtual environment. We conducted a user-study with two users wearing a Head-Mounted-Display in the context of a collaborative FirstPerson-Shooter game. Our three methods were compared with a condition where the physical tracked space was separated into two zones, one per user, to evaluate the impact of each condition on safety, displacement freedom and global satisfaction of users. Results suggest that the ghost avatar and the cylinder grid can be good alternatives to the separation of the tracked space.
\end{abstract}

\section{CCS CONCEPTS}

- Human-centered computing $\rightarrow$ Virtual reality; Collaborative interaction; User studies;

\section{KEYWORDS}

Virtual Reality, Collaborative Virtual Environment

Permission to make digital or hard copies of part or all of this work for personal or classroom use is granted without fee provided that copies are not made or distributed for profit or commercial advantage and that copies bear this notice and the full citation on the first page. Copyrights for third-party components of this work must be honored.

For all other uses, contact the owner/author(s).

VRST '17, November 8-10, 2017, Gothenburg, Sweden

(c) 2017 Copyright held by the owner/author(s).

ACM ISBN 978-1-4503-5548-3/17/11...\$15.00

https://doi.org/10.1145/3139131.3139142

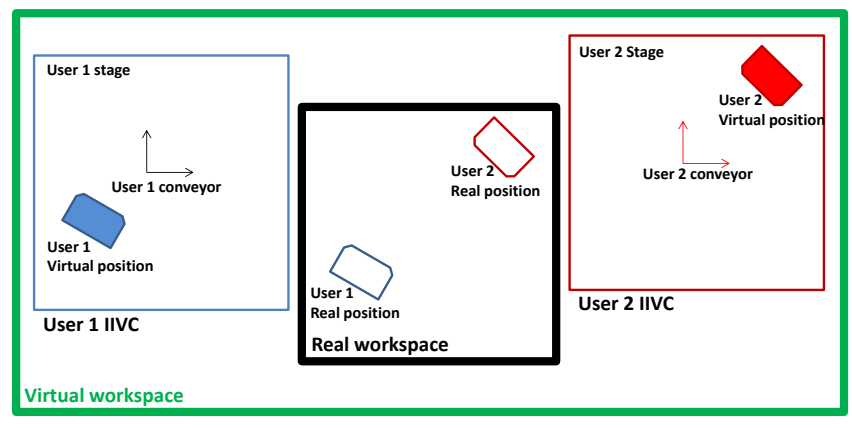

Figure 1: A major problem for a co-located 3D CVE: Spatial desynchronization of two users IIVCs [Fleury et al. 2010] that results from the virtual navigation of both users in the VE. When these two users wear a HMD, they can still perceive each other in the VE but no longer in the real workspace. In that case, we must avoid any possible physical collision between the two users.

\section{ACM Reference Format:}

Jérémy Lacoche, Nico Pallamin, Thomas Boggini, and Jérôme Royan. 2017. Collaborators Awareness for User Cohabitation in Co-located Collaborative Virtual Environments. In Proceedings of VRST '17, Jennifer B. Sartor, Theo D'Hondt, and Wolfgang De Meuter (Eds.). ACM, New York, NY, USA, 9 pages. https://doi.org/10.1145/3139131.3139142

\section{INTRODUCTION}

In the past few years, interest in Virtual Reality (VR) technologies has grown thanks to the appearance of more affordable and more efficient 3D sensors and Head-Mounted Displays (HMDs). Indeed, devices such as the Oculus Rift ${ }^{1}$ or the HTC Vive ${ }^{2}$ provide roomscale VR experiences with hand-based interaction capabilities. Such setup tends to provide experience coming close to system such as CAVEs [Cruz-Neira et al. 1992] but with a lower accessibility threshold. VR technologies have an important number of possible use cases such as gaming, data visualization, learning, ComputerAided Design (CAD), etc. For now, such experiences are mainly single user but can be greatly improved if shared by multiple users.

\footnotetext{
${ }^{1}$ https://www.oculus.com/

${ }^{2}$ https://www.vive.com/
} 
A 3D Collaborative Virtual Environment (3D CVE) is an interactive 3D Virtual Environment (VE) where several users can join to share a collaborative interaction experience [Fleury et al. 2010]. If different users share the same physical workspace, the setup is co-located; however, if they are at different locations, the collaboration is distant. A 3D CVE implies the deployment of methods for maintaining the consistency of the virtual world, for ensuring the communication between different users, and for providing awareness of the other users' activities. For instance, a common awareness method to perceive the other users' activities consists in representing each user by an avatar in the shared virtual world.

This paper is placed in the context of co-located 3D CVEs where each user wears a HMD. Our goal is to solve a particular problem in this kind of situation, which is proper user cohabitation and safety in the shared physical workspace when users can navigate both virtually and physically. This is particularly challenging for the consumer market where users have small tracking spaces and would like to share a virtual experience at home with other users. For the characterization of this issue, we refer to the Immersive Interactive Virtual Cabin (IIVC) [Fleury et al. 2010], which is a model for embedding a user and his/her physical environment into a VE. It is based on two main concepts: the stage is a virtual description of a user's real environment, and the conveyor is the integration frame of the stage into the virtual world that has its own position, rotation, and scale. In a co-located 3D CVE, the stage is the same for every user, as they share the same physical workspace. Each user is commonly represented by an avatar in the VE. In an ideal situation, each user conveyor is exactly the same for every user; therefore, the avatar representation in the VE of each user matches the real position of the user in the stage. However, when the virtual workspace is larger than the real one, navigation interaction techniques must be deployed to let the users explore the entire VE. In that case, each user may move his/her conveyor independently from the others, which would result in a spatial desynchronization of different IIVCs, as shown in Figure 1. Each user would still perceive where the other collaborators are in the VE but no longer where they are related to $\mathrm{him} /$ her in the real world (the stage), as shown in Figure 2. Thus, a metaphor must be deployed to allow the users to avoid each other in the shared real workspace and ensure their safety. That is why, in this paper we propose and compare three visual metaphors in a user study to ensure the safety of collaborators in the shared physical workspace. These methods are compared in an evaluation based on a practical case for consumer market VR applications: a collaborative First-Person-Shooter (FPS) game.

This paper is structured as follows: first we present some related work of collaborative VR and user cohabitation in co-located CVEs. Then, we describe our three awareness methods to provide a proper user cohabitation in co-located CVEs with a HMD-based setup. These three methods are then compared in a user-study with another state of the art method. To finish, we conclude and provide some perspectives for future work.

\section{RELATED WORK}

To ensure safety and a proper user cohabitation between collaborators in a co-located 3D CVE, most methods focus on constraining and adapting the navigation technique. According to Bowman et

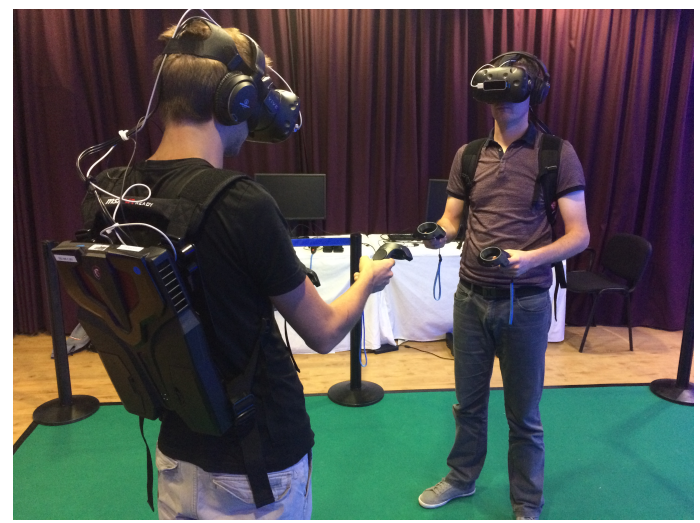

Figure 2: Two users in a co-located 3D CVE. If they virtually navigate in the VE, they must be warned about the position of each other in the physical tracked workspace to avoid possible collisions

al. [Bowman et al. 2004], Navigation is the task of performing the actions that move us from our current location to a new target location. One approach to avoid the desynchronization of the users IIVCs consists of only allowing one user to control the navigation [Beck et al. 2013] [Kulik et al. 2011]. In that case, the conveyors of all users follow the conveyor of one leader. To continue, Chen et al. [Chen et al. 2015] propose a modified version of the human joystick metaphor [McMahan et al. 2012] with an implicit adaptive control that allows safe individual navigation for multiple users in a multi-stereoscopic immersive VE. This approach does not avoid the desynchronization of the users IIVCs, but it ensures to maintain a distance between the different users in the real world. In these different papers, a multi-stereoscopic display system is used; therefore, the users are not isolated from the real world and can still perceive the other users and avoid them when they move in the physical workspace. One of the most relevant solutions has been detailed by Azmandian et al. [Azmandian et al. 2017] and consists of using redirected walking to ensure a safe cohabitation between multiple users wearing HMDs in a shared tracked physical workspace. In a user study, their solution has been preferred compared to a situation where the tracked space was subdivided in one zone per user. However, such approach requires a large tracking space and can be difficult to apply for smaller rooms.

In this paper, instead of constraining the navigation of each user, we propose to adapt the virtual content to warn each user regarding the position of his/her collaborators in the physical workspace. Therefore, we explored the solutions for the representation of physical obstacles and for the representation of users in VR.

In VEs, to avoid collision with real obstacles, the limits of the physical workspace are commonly represented in the virtual world. For instance, in the IIVC model, these limits are represented as semitransparent glasses [Fleury et al. 2010]. In addition, for the same IIVC model, Duval et al. [Duval et al. 2014] represent these limits with a $3 \mathrm{D}$ grid. This is the solution chosen by some commercial solutions such as the HTC Vive and the Oculus Rift. Then, the magic barrier tape [Cirio et al. 2009] displays the boundaries of the physical workspace as a virtual barrier tape that can be pushed to 
navigate in the VE. In the same manner, Cirio et al. [Cirio et al. 2012] presents the virtual companion, which is a virtual bird that protects the user at the limits. All these different methods become visible only when the user gets close to the boundaries of the physical workspace. The HTC Vive can also transform itself into a videosee-through HMD when the user gets close to the boundaries of the physical workspace to perceive eventual obstacles in the real world. To finish, Never Blind VR [Nahon et al. 2015] goes further with an augmented virtuality system that integrates the real world as a point cloud in the VE thanks to a depth sensor. With this system, a user can perceive any obstacle or any other person in the room.

To represent users in a 3DCVE, most approaches are based on humanoid or pseudo-humanoid avatars [Fraser et al. 1999]. With this representation, the activity of each participants can be displayed and perceived in the CVE. The use of pseudo-humanoid embodiments is chosen, as it make all collaborators understand that a user has human-like capabilities in the VE [Hindmarsh et al. 1998]. Avatars can be represented at different levels of realisms from textureless humanoid to realistic male and female avatars [Benford et al. 1995] [Garau et al. 2003]. In some cases, only some body parts of an avatar can be represented and animated. For instance, recent approaches developed for the HTC Vive and Oculus Rift represent a user in the VE as a bust and two hands animated with the tracking from the headset and the controllers. Fully animated avatars can also be deployed with the use of motion capture systems [Mohler et al. 2008]. Some works also propose to animate the avatar's eyes thanks to an eye tracking system to understand the user's intentions better [Steptoe et al. 2008]. In addition, Beck et al. [Beck et al. 2013] proposed a very realistic approach where the appearance of each user is captured and integrated in the 3D CVE in real-time thanks to depth sensors.

\section{THREE AWARENESS METHODS BASED ON TWO DIFFERENT PARADIGMS}

Our approach does not propose to adapt the navigation but to adapt the virtual world content to warn each user about the position of his/her collaborators in the physical workspace to ensure a safe cohabitation. Indeed, solutions such as redirected walking require too large tracking spaces and are not adapted to the consumer market yet. As well, we consider that the subdivision of the tracking space could create situations where each user feels frustrated about the small size of the area where he can physically navigate. That is why, we propose three methods based on current approaches to represent physical obstacles and users in VEs. Indeed, a user is not a simple static obstacle but a dynamic and sometimes unpredictable one.

We propose three metaphors based on two different paradigms. First, we propose to represent a user as a danger to avoid in the physical workspace. Two of our metaphors are based on this paradigm and are described in Sections 3.1.1 and 3.1.2. The second paradigm proposes displaying the physical safe-navigation space in the VE. The third metaphor is based on this paradigm, it is detailed in Section 3.2.

The different metaphors that we propose are only deployed when there is a desynchronization of the users' IIVCs, as shown in Figure 1. Indeed, we consider that without this desynchronization, the representation of each user in the VE (with an avatar, for instance) is enough to avoid physical collisions. Moreover, the different examples that we give are based on a setup where the head and the hands of each user are tracked, but our approaches can be easily extended to cases where we have less or more tracking information. This setup has been chosen because it corresponds to the hardware capabilities available today for the consumer market.

\subsection{Danger awareness}

Our two first approaches are based on the same paradigm: the representation of the collaborator as a danger in the VE to warn the user about a possible collision. These two approaches exploit some related work for the representation of physical obstacles and users in VEs.

3.1.1 The Extended Grid. As detailed in Section 2, the most common obstacle represented in VR is the delimitation of the physical tracked workspace. The 3D grid used to represent this delimitation proposed by Duval et al. [Duval et al. 2014] can today be found in most consumer market applications. For instance, the chaperone system of SteamVR ${ }^{3}$ is based on this solution. Therefore, for a given user, we propose to complete this grid with another grid per collaborator in the shared physical workspace. This technique has the advantage to not totally occlude the user's field of view, as he/she can still see through the grid.

As detailed in Figure 3a, this grid is shaped as a cylinder and progressively appears when the user gets close to his/her collaborator in the physical workspace. This grid takes the same color as the grid that delimits this workspace. For a given collaborator, to place this grid in the VE for each other user, we first compute the tracked positions of this collaborator in the referential of a user's IIVC. The mean value of these positions is used to center the cylinder, and the radius is chosen according to the maximum distance between this center and all the tracked positions. On the up axis, the basis of the cylinder is placed on the floor, and its height is set as $20 \mathrm{~cm}$ on top of the distant user head height. As the position and the shape of the cylinder are updated at each frame, the user can understand the dynamics of the obstacle he faces. The opacity of the grid is chosen according to the distance between the user and the cylinder. It is invisible after a given distance threshold, and the opacity value linearly increases when the user gets close to it. The goal of this progressive fading is to create a security margin between the users while not disturbing the immersion when other users are far away.

3.1.2 The "Ghost" Avatar. As suggested by Azmandian et al. [Azmandian et al. 2017], we propose to warn the user about a possible collision with a collaborator by using a ghost version of his/her avatar representation. The goal is to have a better understanding of which kind of obstacle a user faces and to let him/her better anticipate the dynamics of his/her collaborators movements.

A solution could have been to duplicate the collaborator avatar, place it relatively to the user IIVC, and apply it a semi-transparent visual effect. However, we consider that this solution could create confusion for the user between the avatar that represents the collaborator in the VE and this ghost effect that is supposed to only warn him/her about a possible physical collision. Indeed, this ghost

\footnotetext{
${ }^{3}$ https://steamcommunity.com/steamvr
} 


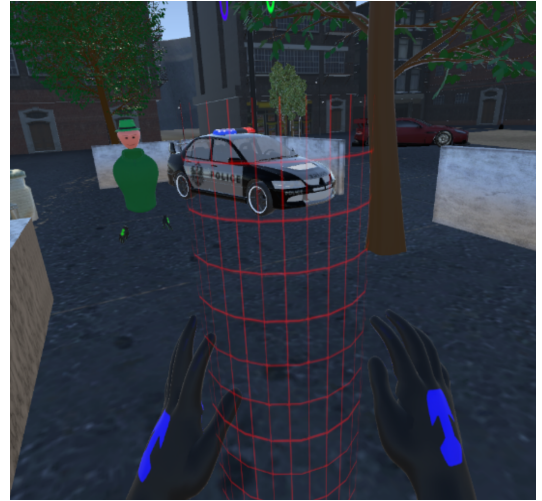

(a)

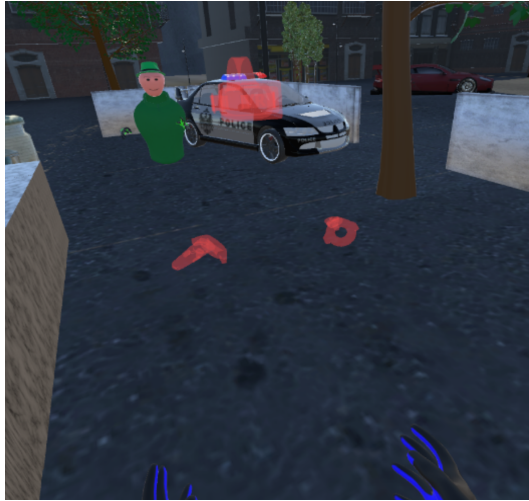

(b)

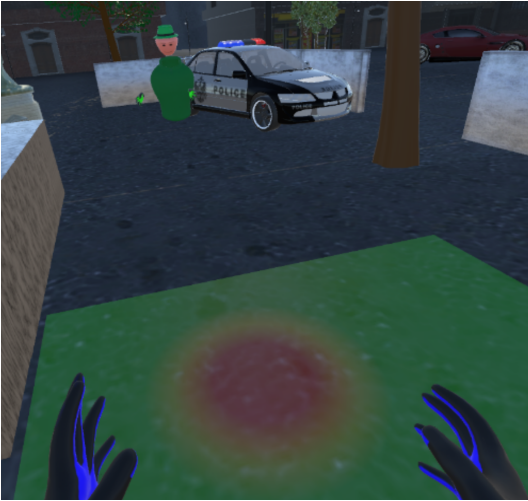

(c)

Figure 3: Three visual metaphors warn users about the position of collaborators in the shared physical workspace (a) First, the physical location of the user is rendered through a grid shaped as a cylinder. (b) Second, we use a ghost representation of the user composed of a HMD model and two controller models to represent his/her head and hands. (c) Lastly, we display on the floor the area where the user can physically go in green and the area taken by the other users in red with a progressive fading between the two zones.

avatar does not need to provide any information about the identity of the collaborator and must keep a neutral representation.

Therefore, we compute the tracked positions (head and hands) of the collaborator in the user's IIVC referential and then, as shown in Figure 3b, we render a HMD model at the head position and controllers models at the hands positions. This representation is enough to make the user aware that he/she is physically close to someone else and does not overload the field of view with useless information. In the same way as the grid, this representation is invisible when a user is far away from his/her collaborator, and the opacity increases when the user gets physically close to his/her collaborator. However, the representation always remains semitransparent to not completely block the field of view of the user.

\subsection{Free-navigation space awareness}

The second paradigm does not warn the user about possible dangers but rather displays the physical safe-navigation space. The goal is to indicate to the user where he can physically navigate.

3.2.1 The Safe Navigation Floor. This technique renders on the ground of the VE where the user can physically navigate in green and where he/she cannot navigate in red, as detailed in Figure 3c. The Safe Navigation Floor is only displayed inside the IIVC of the user to not overload too much the VE. It is always visible when there is a desynchronization between the different users IIVCs.

As shown in Figure 3c, a circle displayed in red corresponds to the position of a collaborator relative to the user IIVC. Similar to the cylinder described in Section 3.1.1, the position of the circle is computed as the center of the tracked positions of the collaborator, and its radius is set as the maximum distance between this center and all the tracked positions. In addition, the shape and the position of this circle are updated at each frame. As detailed on Figure 3c, a progressive linear fading visible in yellow is applied between the safe zone and the zone taken by a collaborator.
This technique has the advantage over our two first methods to only appear on the floor; therefore, most of the time it does not partially occlude the user field of view. Moreover, as it is always visible, the user can always determine the locations of his/her partners to plan his/her next physical movements. However, as this plane is always displayed, it can affect the realism of the VE.

\section{EVALUATION}

\subsection{Experimentation description}

We ran a user experiment to evaluate the efficiency of our different approaches compared to a separation of the tracked space into two different areas, one per user, as proposed in [Azmandian et al. 2017]. Therefore, the different conditions that we compared were as follows: Extended Grid (EG), Ghost Avatar (GA), Safe Navigation Floor (SNF), and Separated tracked spaces (STS). We compared the different methods within a simple collaborative First-Person-Shooter game (FPS). This kind of application has been chosen because it requires both virtual and physical displacements and coordination between multiple users. Moreover, it suits well the possible uses of such approaches: people at home who want to share a co-located collaborative VR experience in a small tracked space.

Our experimentation panel consisted of 19 groups of 2 persons, thus 38 subjects aged from 14 to 59 (age: $M=32, S D=11$ ). There were 29 males and 9 females. Most of them were inexperienced in VR, but the majority of subjects was used to 3D games. The subjects have various backgrounds: students, R\&D engineers, managers, and assistants.

Application. As detailed, we used a simple FPS in our experiment to compare our different conditions. The game mechanics and interactions have been chosen to be easily understood by users, even the novice ones. Moreover, the application has been designed to make users navigate both physically and virtually. A simple virtual representation of users was chosen. It was based on a partial avatar 


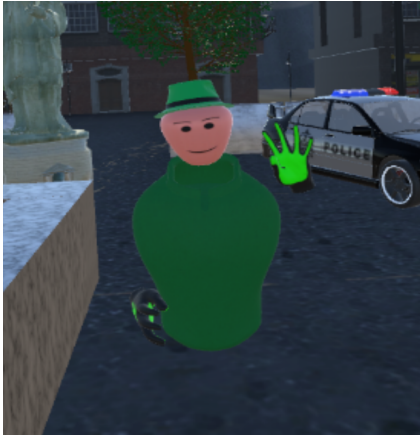

(a)

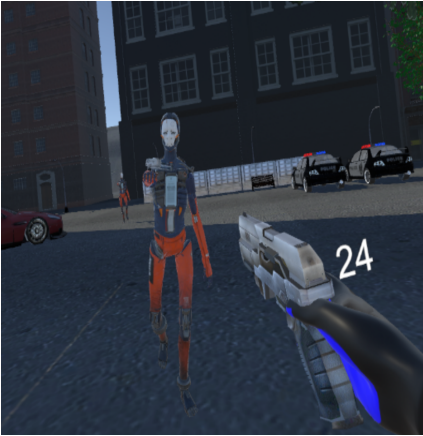

(b)
Figure 4: The different metaphors were compared with a FPS game. (a) User were represented in the shared VE with an avatar. (b) The goal of the game was to shoot at robots and avoid their projectiles. Most assets were taken from the Web such as the Adam character from the Unity3D tech demo ${ }^{5}$.

composed of a head linked to a bust and of two separated hands, as shown in Figure 4a. One avatar was blue, while the other avatar was green.

At the beginning of the game, both users were situated on the center of the small town. Robots spawned randomly around the map, users had to eliminate them and avoid their projectiles as shown in Figure $4 \mathrm{~b}$. These projectiles were slow and visible enough to be properly avoided by users. We deployed single user interactions and collaborative ones:

- With his/her dominant hand, the user held a simple gun as shown in Figure $4 \mathrm{~b}$. The trigger of the controller was used to shoot. One bullet was enough to destroy a robot. To challenge the users, the shooting mechanism was temporary blocked when he/she got touched by multiple projectiles. Moreover, ammunitions were limited but two mechanisms were deployed to reload:

- With his/her non-dominant hand the user could also collect ammo item on the floor with a hand-based selection technique.

- A collaborative interaction was used to fully reload the weapon. Both users had to clap their non-dominant hands. To make the interaction intentional, they had to press the trigger of the controller to close the virtual hand.

- For the virtual navigation, a ray based teleportation technique was used. With his/her non-dominant hand, the user had to point on the floor while pressing a button to indicate his/her destination. To not further promote virtual navigation over physical movements, the teleportation was only possible every 10 seconds.

The application was implemented with Unity3D and with the SteamVR plugin. A software layer based on Photon Engine ${ }^{4}$ was used to handle the synchronization of the VE and the collaborative interactions. Most of assets were downloaded from the Web and from the Unity Assets Store.

\footnotetext{
${ }^{4}$ https://www.photonengine.com/en-US/Photon
}

Hardware. Each user was equipped with a HTC Vive with its two controllers and with a MSI VR One backpack PC ${ }^{6}$. A backpack based configuration has been chosen to remove the influence of the wire on physical movements of users, as shown in Figure 2. Moreover, we strongly believe that the next generation of HMDs for the consumer market will be wireless. With this setup, the application was running at $90 \mathrm{fps}$.

The dimensions of the tracked space was set to $3 \times 3$ meters. These dimensions have been chosen, because it can correspond to the available space of a common consumer setup. For the separated tracked space condition, this space was separated into two zones of $1.4 \times 3$ meters with a $20 \mathrm{~cm}$ safe area between the two zones to maximize the safety of users.

Procedure. Each group of two users tested the four conditions in four game sessions of 3 minutes. In each session, the effect deployed for each user was the same. We applied a counterbalancing design to change the order of the tested conditions for each group. Users had 5 minutes of break between sessions to complete a questionnaire and to rest. During this break, the two users could not talk to each other. For EG and GA conditions, the same parameters for the fading security margin were chosen, they started to appear at 1 meter of physical distance from the other user and became completely visible at a distance of 0.5 meter. For all conditions, a virtual 3D grid was used to delimit the physical tracked space. In addition, this grid was only visible when the user got close to it. The color of this grid was set in red, the same color as the effects detailed in Section 3 to ensure overall coherence. For the STS condition, each user was placed in one of the two zones which was virtually delimited with this 3D grid. Collisions could occur if a user crossed his/her grid and left his/her zone.

Collected Data. Between each session, a subjective questionnaire was proposed to each user. In this questionnaire, participants had to grade the tested condition using a Likert scale, from -3 (lowest appreciation) to 3 (highest appreciation) on questions based on multiple categories: feeling of security, displacement freedom, and global appreciation. At the end of all sessions, users were asked to rank each condition from 0 to 10 on three criteria: global satisfaction, estheticism and efficiency.

Moreover, we also collected quantitative data during the experiment. First, physical collisions events were counted by the experimenter. Second, all virtual and physical positions of users HMDs and controllers were collected to compute interpersonal distances and physical movements. Last, game events have been collected to deduce performances data.

Hypotheses. We established three main hypotheses based on the criteria we wanted to evaluate: safety, displacement freedom, and global appreciation. Both subjective and objective results will be used to verify these hypotheses:

- H1: The safety of users will be better with the STS condition.

H1.1: Less collisions will happen with the STS condition, and the physical distance between the two users will remain higher with this condition.

\footnotetext{
${ }^{5}$ https://unity3d.com/fr/pages/adam

${ }^{6}$ http://vr.msi.com/Backpacks/vrone
} 


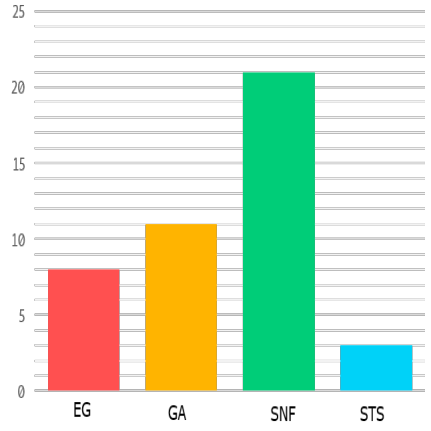

(a)

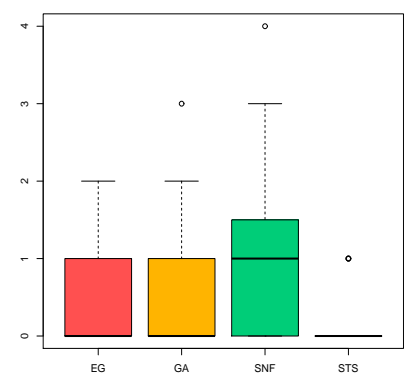

(b)

Figure 5: (a) The total number of collisions per condition (EG, GA, SNF, and STS) in all sessions of 3 minutes per condition. (b) Boxplot of collisions per condition.

H1.2: Users will feel safer and less afraid of possible collisions with the STS condition.

- H2: People will be more free in their physical movements in EG, GA, and SNF conditions than in the STS condition.

H2.1: Users will physically move more in these three conditions than in the STS condition.

H.2.2: Users will feel more free in their physical movement and less frustrated.

- H3: Users will globally prefer being in a shared tracked space with one of our metaphors deployed (EG, GA, SNF) than being constrained to a smaller physical space (STS)

\subsection{Results}

Safety of users. To validate H1, we first analyzed the number of physical collisions between users reported by the experimenter and analyzed the physical distances between users during the simulation. Figures $5 \mathrm{a}$ and $5 \mathrm{~b}$ illustrate the collision numbers results. First, it is important to report that nobody has been hurt by one of these collisions during the experimentation. We performed an analysis of variance (ANOVA) on these results, and we found that the deployed method had a significant impact on the number of collisions $\left(F_{37,111}=9.57, p<0.001\right)$. Then, for comparing each pair of conditions, we performed a post hoc t-test with Bonferroni correction. We found a significant difference between SNF and STS conditions $(p<0.001)$, between SNF and EG conditions $(p<0.01)$, and between SNF and GA conditions $(p<0.01)$. The number of collisions was significantly higher with SNF condition $(M=2.1, s d=1.20)$ than with STS $(M=0.3, s d=0.37)$, than with $\mathrm{EG}(M=0.8, s d=0.69)$, and than with $\mathrm{GA}(M=1.1, s d=0.84)$. We did not find any significant result between EG and STS conditions and between GA and STS conditions. Regarding physical distances between users, we computed the physical distance between the heads of the two users twice per second. The results are illustrated in Figure 6. An ANOVA demonstrated a significant impact of the condition on these results $\left(F_{18,54}=9.80, p<0.001\right)$. After post-hoc t-tests with Bonferroni correction, we found a significant difference for the distance between users, between STS

and EG conditions $(p<0.001)$, between STS and GA conditions $(p<0.001)$ and between STS and SNF conditions $(p<0.01)$. Indeed, the distance between users remained higher with STS condition $(M=1.8, s d=0.34)$, than with EG $(M=1.58, s d=0.44)$, than with $\mathrm{GA}(M=1.55, s d=0.42)$, and than with $\operatorname{SNF}(M=1.63, s d=0.41)$. No significant difference was found between EG, GA and SNF conditions.

To continue, we also observed subjective results, the answers to two questions asked between sessions. The first one was "I was able to move physically safely" and the second one was "It was easy to anticipate the movements in the physical world of my partner and

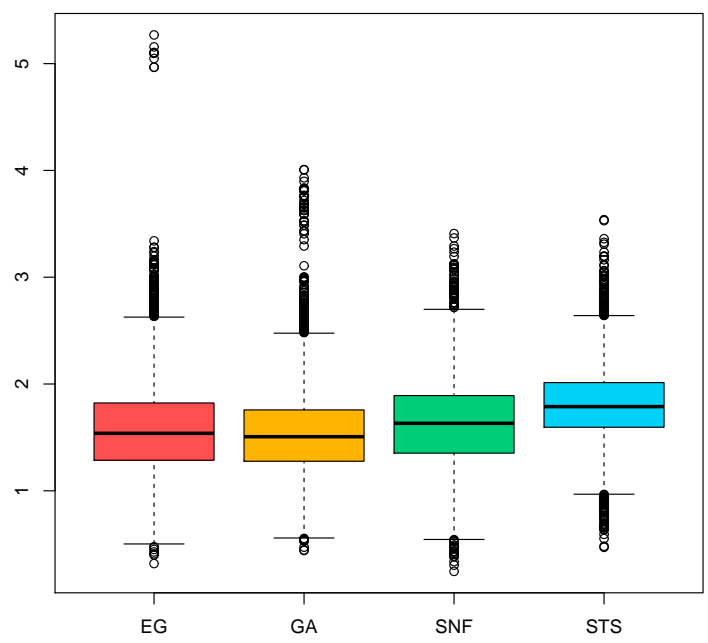

Figure 6: Boxplot for the physical distance between the heads of the two users collected twice per second.

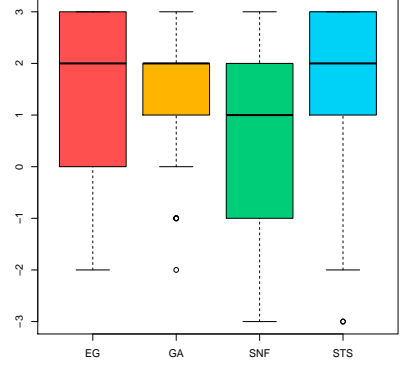

(a)

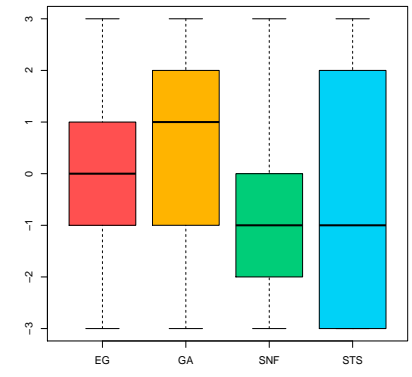

(b)
Figure 7: (a) Boxplot of subjective results for the criteria of safety during physical movements. (b) Boxplot of subjective results for the capacity to easily anticipate the physical movements of the other user and avoid him/her. 
avoid them". The results for these two questions are shown in Figures $7 \mathrm{a}$ and $7 \mathrm{~b}$. The results of these two questions were analyzed using the Friedman chi-squared test and Wilcoxon pair-wise tests with Bonferroni correction. A significant impact of the condition on the results of this questions has been found $\left(\tilde{\chi}^{2}=9.98, p<0.05\right)$. More precisely, a significant difference was found between EG and SNF conditions $(p<0.01)$ and between GA and SNF conditions $(p<0.01)$. EG and GA were considered safer by users during physical displacements than SNF metaphor. No significant difference was found between STS conditions and our three effects on this criteria. Regarding anticipation, a significant difference between the conditions was also found $\left(\tilde{\chi}^{2}=17.26, p<0.001\right)$. Users significantly considered it to be easier to anticipate the other physical movements with the EG condition compared to the SNF condition $(p<0.001)$, with the GA condition compared to the SNF condition $(p<0.001)$, and with the GA condition compared to the STS condition $(p<0.01)$.

Displacement Freedom of users. To verify H2, we first analyzed the quantitative results, which are the distance covered by each user in the physical world extracted from the heads position recorded every frame, and the number of teleportation (virtual navigation) performed by each user. Then, we also analyzed the subjective feeling of freedom expressed by users in the questionnaires.

The displacements in the physical world and the number of teleportation have been analyzed using an ANOVA. We did not find any significant differences regarding the distance covered by users in the physical world. It means that whatever the condition, users walked approximately the same distance in the physical tracked space. Regarding virtual navigation, no significant difference was found for the number of teleportations.

One question asked between sessions concerned the feeling of displacement freedom, this question was: Did you sometimes feel frustrated to not be able to physically move?". For this question, we performed a Friedman chi-squared test and we did not find any significant impact of the condition on the results. However, we observed that multiple users had the same reaction at the beginning of the application with the STS condition. Indeed, users told us that they felt "inside a cage" or "inside a prison". Some of these reactions can also be found in the comments sections of the questionnaires. Nevertheless, these reactions are not enough to make any precise conclusion on the feeling of displacement freedom.

Global appreciation of users. To validate H3, we analyzed the results of the question about global satisfaction asked between sessions, and of the grades given by users at the end of the experimentation for each condition on three criteria: experience quality, estheticism, and efficiency of the deployed metaphor. We also analyzed the differences of performances between the conditions for our application.

Regarding the feeling of satisfaction, the question asked to users between sessions was "How much do you feel satisfied about your experience in the Virtual Environment". A Friedman chi-squared test confirmed a significant impact of the conditions on the results of this question $\left(\tilde{\chi}^{2}=16.80, p<0.001\right)$. Then, after Wilcoxon pair-wise tests with Bonferroni correction, we found a significant difference between GA and STS conditions $(p<0.01)$, and between GA and SNF conditions $(p<0.001)$. Indeed, users expressed a better

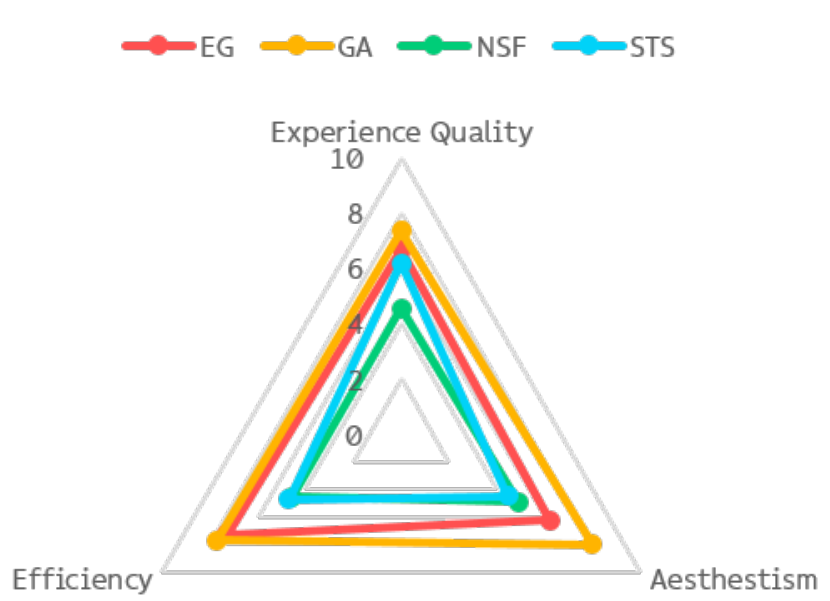

Figure 8: Results for the grades obtained by the four conditions on experience quality, estheticism, and efficiency

Table 1: Statistical results for the comparison of the grades obtained by the four conditions on experience quality, estheticism, and efficiency.

\begin{tabular}{|c|c|c|c|c|}
\hline \multicolumn{2}{|c|}{$\begin{array}{l}\text { Post hoc T-Tests with } \\
\text { Bonferroni correction }\end{array}$} & \multirow[b]{2}{*}{ GA } & \multirow[b]{2}{*}{ SNF } & \multirow[b]{2}{*}{ STS } \\
\hline $\begin{array}{r}D f=35 \\
N S=\text { No Signific }\end{array}$ & $\begin{array}{l}\text { Ue } \\
\text { ifference }\end{array}$ & & & \\
\hline \multirow{3}{*}{$\begin{array}{l}\text { Experience } \\
\text { Quality }\end{array}$} & EG & NS & $\begin{array}{c}p<0.001 \\
t=4.95\end{array}$ & NS \\
\hline & GA & & $\begin{array}{l}p<0.001 \\
t=-5.89\end{array}$ & NS \\
\hline & SNF & & & $\begin{aligned} P & <0.01 \\
t & =-3.0824\end{aligned}$ \\
\hline \multirow{3}{*}{ Aesthetic } & EG & $\begin{array}{l}P<0.001 \\
t=-3.93\end{array}$ & NS & NS \\
\hline & GA & & $\begin{array}{l}P<0.001 \\
t=-5.99\end{array}$ & $\begin{array}{l}P<0.001 \\
t=-6.63\end{array}$ \\
\hline & SNF & & & NS \\
\hline \multirow{3}{*}{ Efficiency } & EG & NS & $\begin{array}{l}P<0.001 \\
t=4.66\end{array}$ & $\begin{array}{l}\mathrm{P}<0.01 \\
\mathrm{t}=3.42\end{array}$ \\
\hline & GA & & $\begin{array}{c}\mathrm{P}<0.001 \\
\mathrm{t}=-6.3663\end{array}$ & $\begin{array}{c}P<0.001 \\
t=-4.5\end{array}$ \\
\hline & SNF & & & NS \\
\hline
\end{tabular}

satisfaction with the GA condition $(M=2.47, s d=0.69)$ compared to the STS condition $(M=1.92, s d=1.0)$ and the SNF condition $(M=1.71, s d=1.27)$. No significant different between GA and EG conditions was found for this question.

For the grades given at the end of the experimentation, we performed an ANOVA and found significant differences for all criteria: experience quality $\left(F_{35,105}=11.771, p<0.001\right)$, estheti$\operatorname{cism}\left(F_{35,105}=15.632, p<0.001\right)$, and efficiency of the deployed metaphor $\left(F_{35,105}=14.338, p<0.001\right)$. We show the results on Figure 8 and the post-hoc analysis in Table 1. Regarding experience quality, SNF condition obtained significantly lower grades compared to the other ones. No significant difference was found between the other effects for this criteria. To continue, regarding estheticism of the deployed metaphors, according to users, the 
GA condition was significantly more esthetic than the three other conditions. No significant difference was found between the other effects for this criteria. Lastly, regarding global efficiency of the deployed metaphor, according to users, EG and GA conditions were considered significantly more efficient than the SNF condition and the STS condition. However, no significant difference was found between GA and EG effects for this criteria. We also tried to compare the performances obtained by users with different conditions. Therefore, we analyzed the following quantitative data:

- The number of times the user is touched by a projectile

- The number of times the user destroys an enemy

For these two kinds of data, we did not find any significant difference between the different conditions, so we can say that the deployed metaphor for avoiding themselves did not affect the users' performances in our application.

For our three metaphors, EG, GA, and SNF, users were also asked if they felt disturbed by one of these metaphors and if sometimes they confused the user's avatar with the virtual representation of his/her physical location. For these two questions, we did not find any significant difference. Users tended to answer that they did not feel disturb by the deployed metaphor: $\mathrm{EG}(M=-0.97, s d=1.97)$, GA $(M=-1.03, s d=2.01)$, and SNF $(M=-0.71, s d=2.18)$. As well, they tended to answer that they did not confuse the virtual and the physical representations: EG $(M=-1.5, s d=1.8)$, GA $(M=-1.13, s d=2.06)$, and SNF $(M=-1.18, s d=2.02)$.

\subsection{Discussion}

Our hypotheses were not all verified.

First, regarding safety of users, for H1, as planned, the physical distance between users remained higher when they were separated into two different zones. However, for the number of collisions, the difference was not significant with the Extended Grid and with the Ghost Avatar metaphors compared to the Separated Tracked Spaces condition. It means that even if users were globally closer with these two metaphors, it did not produce significantly more collisions. This observation tends to demonstrate the efficiency of these two metaphors on safety of users. Thus, H1.1 was not completely verified. In addition, in 38 sessions of 3 minutes, 3 collisions happened when users were separated into two different zones, which demonstrates that this method was not completely safe either. To continue, the Safe Navigation Floor produced significantly more collisions than the three other effects. Many users told us that this metaphor forced them to always look at the floor and that it was difficult to conciliate this task with the game. We really believe that this was the main reason of the inefficiency of this method for ensuring the safety of users. Globally, the subjective results match these quantitative results; therefore, H1.2 was not verified. Indeed, users only felt less safe with the Safe Navigation Floor than with the three other methods. However, users did not express a safety difference between the other effects. It confirms the collisions results. Lastly, we also analyzed the subjective results regarding the capacity of each user to anticipate the physical movements of his/her partner to avoid him/her. Our first thought was that the Ghost Avatar would be the best metaphor for this criteria, because it provides precise information about the user's head and hands positions. The results analysis provided in Section 4.2 confirms that the Ghost Avatar was significantly considered as the best metaphor for this criteria compared to the three other methods.

Second, for displacement freedom, H2 was not verified. Indeed, users did not physically move more with one condition or with another one. In addition, the teleportation interaction was globally used the same number of times for all conditions. These results were confirmed by the subjective questionnaires where users did not express any differences of frustration during physical movements depending on the condition. Even if some users told us about this frustration or wrote it in the comments section of the questionnaire, it was not confirmed by the statistical results; thus, we cannot make any conclusions. We were surprised about these results, because we really thought that being in a larger space would make the users move more freely, especially since there were differences between conditions for the safety criteria. We think that it could be interesting to investigate more on this criteria with another experiment based on another application that requires more physical movements.

Third, for global appreciation and quality of experience, $\mathbf{H} \mathbf{3}$ was verified. Indeed, users globally appreciated more being in a shared tracked space with the Ghost Avatar or with the Extended grid metaphor compared to be constrained into a smaller physical space. We found the same results for estheticism and perceived efficiency criteria, but the differences for the quality of experience were less significant. Nevertheless, for these criteria, the condition that obtained the worst results was the Safe Navigation Floor. Indeed, it has been globally less appreciated, and considered less esthetic and efficient than the three other conditions. We think that these results can be explained by the increased number of collisions with this metaphor and because it constrained users to always look at the floor. Moreover, users tended to say that they did not feel confused between the virtual representation of a user and one of our awareness methods and that they did not feel disturbed by one of these metaphors during their experience. As detailed in Section 4.2, we did not observe significant differences of performances between the four conditions. We think that these results match the results obtained for displacement freedom. Indeed, as users did not move more depending on the condition, we believe that their behavior, and therefore their performances in the game, did not differ either.

Our two metaphors, the Extended Grid and the Ghost Avatar obtained good safety results and were globally more appreciated than the two other conditions. Therefore, we think that they could be used as an alternative to the separation of the tracked space into multiple zones. Nevertheless, globally, the Safe-Navigation-Floor was the metaphor that obtained the lower results, in particular for the safety and global satisfaction criteria. Improvements of this metaphor should be investigated to improve its efficiency. For instance, it could be combined with a danger awareness metaphor such as the Ghost Avatar or the Extended grid. Indeed, the SafeNavigation-Floor would continuously inform the users where he can physically go and a danger awareness metaphor would warn him/her about possible physical collisions. To continue, regarding collisions, all our methods have the same drawback, they only appear in the field of view of the user. For instance, if both users move backwards, our effects do not prevent any physical collision. However, the same kind of collisions can happen even without a 
desynchronization of IIVCs with an avatar-based user representation. Other kinds of effects should be investigated to solve this issue, for instance, effects based on peripheral visual effects or based on spatialized sound. Lastly, we think that the different metaphors should be compared with another application to validate our results and investigate more the displacement freedom criteria. In addition, more tests could also be needed with more than two users.

\section{CONCLUSION AND FUTURE WORK}

In this paper, we propose three metaphors to ensure the safety of users in a co-located collaborative VE when users can move differently in the virtual space and in the physical space. We define this problem as a desynchronization of multiple users' Immersive Interactive Virtual Cabins (IICVs) according to the model proposed by Fleury et al. [Fleury et al. 2010]. The Ghost Avatar is a simplified version of the user representation that appears when a user gets physically close to him/her. The Extended Grid that appears in the same case is a cylinder that encompasses the physical location of a user. The Safe Navigation Floor always displays on the floor where a user can physically go in green and where he cannot in red based on the other users physical positions. We compared in an experiment these three metaphors with a condition based on the separation of the physical tracked space into one zone per user. Results on safety of users and global satisfaction demonstrate that the Ghost Avatar and the Extended Grid can be good alternatives to the separation of the tracked space.

Our metaphors could also be used for two users sharing the same physical space but not the same Virtual Environment. For instance, it could be two users playing two different games in the same room as long as a software layer can have access to the physical location of each user in each application. In addition, they could also be extended to other dynamic objects such as robots or animals if they can be tracked in the physical space.

Our future work will be focused on other methods to solve the same kind of issues. First we plan to combine the Safe Navigation Floor metaphor with the Extended Grid or with the Ghost Avatar to combine their advantages. Second, the experimentation made us realize some particular cases of collisions that still happen with metaphors rendered in the 3D world, for instance, when users move backwards. We plan to experiment effects based on peripheral visual effects and on spatialized sound to improve the safety of users in these cases. Solutions based on a video see-through HMD or on augmented virtuality could also be experimented. Additional experiments with other applications and with more users are also planned as future work. Other use cases could be explored such as collaborative architectural design and virtual training scenarios.

\section{ACKNOWLEDGEMENTS}

We wish to thank Ific Goudé for his help during the experimentation, Kévin Le Duff for the modeling, and Clément Derré for his advices on the game mechanics.

\section{REFERENCES}

Mahdi Azmandian, Timofey Grechkin, and Evan Suma Rosenberg. 2017. An evaluation of strategies for two-user redirected walking in shared physical spaces. In Virtual Reality (VR), 2017 IEEE. IEEE, 91-98.
Stephan Beck, Andre Kunert, Alexander Kulik, and Bernd Froehlich. 2013. Immersive group-to-group telepresence. IEEE Transactions on Visualization and Computer Graphics 19, 4 (2013), 616-625.

Steve Benford, John Bowers, Lennart E Fahlén, Chris Greenhalgh, and Dave Snowdon. 1995. User embodiment in collaborative virtual environments. In Proceedings of the SIGCHI conference on Human factors in computing systems. ACM Press/AddisonWesley Publishing Co., 242-249.

Doug A. Bowman, Ernst Kruijff, Joseph J. LaViola, and Ivan Poupyrev. 2004. 3D User Interfaces: Theory and Practice. Addison Wesley Longman Publishing Co., Inc., Redwood City, CA, USA.

Weiya Chen, Nicolas Ladeveze, Céline Clavel, Daniel Mestre, and Patrick Bourdot. 2015. User cohabitation in multi-stereoscopic immersive virtual environment for individual navigation tasks. In 2015 IEEE Virtual Reality (VR). IEEE, 47-54.

Gabriel Cirio, Maud Marchal, Tony Regia-Corte, and Anatole Lécuyer. 2009. The Magic Barrier Tape: A Novel Metaphor for Infinite Navigation in Virtual Worlds with a Restricted Walking Workspace. In Proceedings of the 16th ACM Symposium on Virtual Reality Software and Technology (VRST '09). ACM, New York, NY, USA, $155-162$.

Gabriel Cirio, Peter Vangorp, Emmanuelle Chapoulie, Maud Marchal, Anatole Lécuyer, and George Drettakis. 2012. Walking in a cube: Novel metaphors for safely navigating large virtual environments in restricted real workspaces. IEEE Transactions on Visualization and Computer Graphics 18, 4 (2012), 546-554.

Carolina Cruz-Neira, Daniel J. Sandin, Thomas A. DeFanti, Robert V. Kenyon, and John C. Hart. 1992. The CAVE: Audio Visual Experience Automatic Virtual Environment. Commun. ACM 35, 6 (June 1992), 64-72.

Thierry Duval, Thi Thuong Huyen Nguyen, Cédric Fleury, Alain Chauffaut, Georges Dumont, and Valérie Gouranton. 2014. Improving awareness for 3D virtual collaboration by embedding the features of users' physical environments and by augmenting interaction tools with cognitive feedback cues. Fournal on Multimodal User Interfaces 8, 2 (2014), 187-197.

Cédric Fleury, Alain Chauffaut, Thierry Duval, Valérie Gouranton, and Bruno Arnaldi. 2010. A Generic Model for Embedding Users' Physical Workspaces into Multi-Scale Collaborative Virtual Environments. In ICAT 2010 (20th International Conference on Artificial Reality and Telexistence). Adelaide, Australia.

Mike Fraser, Steve Benford, Jon Hindmarsh, and Christian Heath. 1999. Supporting awareness and interaction through collaborative virtual interfaces. In Proceedings of the 12th annual ACM symposium on User interface software and technology. ACM, $27-36$.

Maia Garau, Mel Slater, Vinoba Vinayagamoorthy, Andrea Brogni, Anthony Steed, and M. Angela Sasse. 2003. The Impact of Avatar Realism and Eye Gaze Control on Perceived Quality of Communication in a Shared Immersive Virtual Environment. In Proceedings of the SIGCHI Conference on Human Factors in Computing Systems (CHI '03). ACM, New York, NY, USA, 529-536.

Jon Hindmarsh, Mike Fraser, Christian Heath, Steve Benford, and Chris Greenhalgh. 1998. Fragmented interaction: establishing mutual orientation in virtual environments. In Proceedings of the 1998 ACM conference on Computer supported cooperative work. ACM, 217-226.

Alexander Kulik, André Kunert, Stephan Beck, Roman Reichel, Roland Blach, Armin Zink, and Bernd Froehlich. 2011. C1x6: a stereoscopic six-user display for co-located collaboration in shared virtual environments. ACM Transactions on Graphics (TOG) 30, 6 (2011), 188 .

Ryan P McMahan, Doug A Bowman, David J Zielinski, and Rachael B Brady. 2012. Evaluating display fidelity and interaction fidelity in a virtual reality game. IEEE Transactions on Visualization and Computer Graphics 18, 4 (2012), 626-633.

Betty J Mohler, Heinrich H Bülthoff, William B Thompson, and Sarah H Creem-Regehr. 2008. A full-body avatar improves egocentric distance judgments in an immersive virtual environment. In Proceedings of the 5th symposium on Applied perception in graphics and visualization. ACM, 194.

David Nahon, Geoffrey Subileau, and Benjamin Capel. 2015. âĂIJNever Blind VRâĂİ enhancing the virtual reality headset experience with augmented virtuality. In Virtual Reality (VR), 2015 IEEE. IEEE, 347-348.

William Steptoe, Robin Wolff, Alessio Murgia, Estefania Guimaraes, John Rae, Paul Sharkey, David Roberts, and Anthony Steed. 2008. Eye-tracking for avatar eyegaze and interactional analysis in immersive collaborative virtual environments. In Proceedings of the 2008 ACM conference on Computer supported cooperative work. ACM, 197-200. 